\title{
An approach to pleomorphic sarcomas: can we subclassify, and does it matter?
}

\author{
John R Goldblum \\ Department of Anatomic Pathology, Cleveland Clinic, Cleveland, OH, USA
}

\begin{abstract}
The term malignant fibrous histiocytoma (MFH) has been supplanted by undifferentiated pleomorphic sarcoma (UPS). Even now, however, a number of pleomorphic neoplasms are classified as UPSs when in fact at least a subgroup of these can be more precisely classified as a pleomorphic sarcoma with a specific line of differentiation. Still others are pseudosarcomas, most commonly sarcomatoid carcinomas. This review will discuss historical aspects of MFH/UPS as well as provide an approach to the pleomorphic malignant neoplasm with a discussion of useful ancillary techniques in the evaluation of such cases.

Modern Pathology (2014) 27, S39-S46; doi:10.1038/modpathol.2013.174
\end{abstract}

Keywords: malignant fibrous histiocytoma; pleomorphic sarcoma; sarcomatoid carcinoma; undifferentiated pleomorphic sarcoma

The concept of malignant fibrous histiocytoma $(\mathrm{MFH})$ has undergone significant change over the past five decades. The term was first introduced in 1963 to refer to a group of soft tissue tumors characterized by a storiform or cartwheel-like growth pattern, which were believed to be derived from histiocytes on the basis of early tissue culture studies demonstrating ameboid movement and phagocytosis of explanted tumor cells. ${ }^{1,2}$ However, ultrastructural studies both endorsed and refuted the histiocytic origin of these tumors. With the advent of immunohistochemistry and the accessibility of numerous monoclonal antibodies directed against various structural proteins of specific cell types, the phenotype of this tumor was shown to be more closely aligned with a fibroblast than a histiocyte. ${ }^{3-6}$ Furthermore, many, but not all, lesions labeled as 'malignant fibrous histiocytoma' could, upon close scrutiny, be subclassified as lineage-specific sarcomas, an observation that led some to question the existence of $\mathrm{MFH}$ as a distinct entity. ${ }^{7}$ The extent to which such lesions can be subclassified as sarcomas of alternative type is, in large part, dependent on definitional criteria and the number of ancillary studies a pathologist is willing to bring to bear on the evaluation of a pleomorphic sarcoma. There is still no general agreement as to what percentage of

Correspondence: Dr JR Goldblum, MD, Department of Anatomic Pathology, Cleveland Clinic, 9500 Euclid Avenue L25, Cleveland, OH 44195, USA.

E-mail: goldblj@ccf.org

Received 14 June 2013; revised 28 June 2013; accepted 29 June 2013 pleomorphic sarcomas, when subjected to rigorous evaluation, remain unclassified. These discrepancies, nonetheless, underscore the fact that the criteria by which a pleomorphic tumor is provisionally labeled as an undifferentiated pleomorphic sarcoma (UPS/MFH) as well as the criteria by which some are reclassified differ from institution to institution.

Whatever the true incidence of this lesion, there is agreement that the term MFH should be used synonymously with UPS which, by a combination of sampling and immunohistochemistry, shows no definable line of differentiation and by electron microscopy manifests fibroblastic/myofibroblastic features. ${ }^{8}$ At this point, we use the term UPS in our diagnostic reports, but also state that this is synonymous with so-called MFH in parentheses so as to avert any misunderstanding with clinicians who continue to be familiar with that term.

Whenever I encounter a soft tissue neoplasm with a 'MFH-like' pattern, I consider several broad possibilities before concluding that the lesion is in fact an UPS. First, I consider whether the lesion in question is some type of pleomorphic sarcoma with a specific line of differentiation that can be identified through light microscopy and/or immunohistochemistry. The rationale behind attempting to more precisely classify a pleomorphic sarcoma is discussed below. Second, I consider the possibility that the lesion could be a component of a dedifferentiated sarcoma, particularly when dealing with a sarcoma in the retroperitoneum. Thorough sampling is often required in order to recognize the low-grade sarcoma from which the dedifferentiated 'MFH-like' 
areas arose. Importantly, I also want to exclude the possibility that the lesion is a non-mesenchymal neoplasm. The most common consideration is that of a sarcomatoid carcinoma, particularly when the lesion arises on a mucosal surface, skin, or within a parenchymal organ. Other considerations might include (depending upon site and other clinical factors) sarcomatoid mesothelioma, melanoma, and even anaplastic lymphoma. Finally, if all of these can be excluded, then one can arrive at the conclusion that the lesion is a UPS (Figure 1).

\section{Pleomorphic sarcoma with a specific line of differentiation}

A variety of pleomorphic sarcomas may have areas that resemble UPS. In some cases, determining the specific line of differentiation may rely on random sampling of a small area within a large tumor. Although a specific type of pleomorphic sarcoma may be suggested by histologic features, immunohistochemical stains are often required to confirm the diagnosis. Although it could be argued that subtyping pleomorphic sarcomas is nothing more than an academic exercise, there is some evidence to suggest that pleomorphic sarcomas with myogenic differentiation are more clinically aggressive than those without myogenic differentiation ${ }^{9-11}$ (see below).

The only criterion for rendering a diagnosis of pleomorphic liposarcoma is the recognition of multivacuolated pleomorphic lipoblasts (Figure 2). The major difficulty in such cases is separating pleomorphic sarcomas that infiltrate fat and isolate individual cells from those with true lipoblasts. Pleomorphic leiomyosarcoma is composed of cells with distinct cytoplasmic eosinophilia (Figure 3). At least focally, most cases have areas with a fascicular arrangement and cells with blunt-ended nuclei with a perinuclear vacuole and deeply eosinophilic cytoplasm. Pleomorphic rhabdomyosarcoma

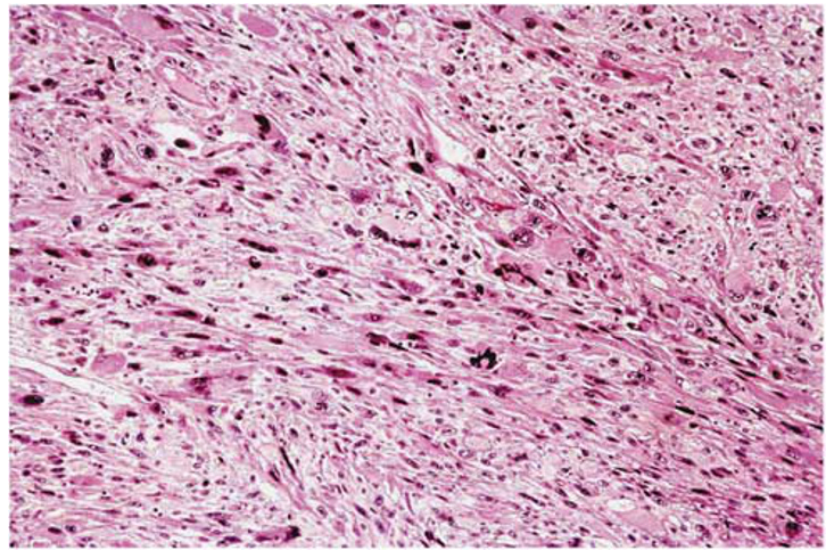

Figure 1 This high-grade pleomorphic malignant neoplasm was ultimately classified as an undifferentiated pleomorphic sarcoma, a diagnosis of exclusion. is recognized by the presence of large cells with eosinophilic cytoplasm and cross striations (Figure 4), which can be confirmed by the immunohistochemical demonstration of skeletal muscle differentiation (desmin, MyoD1, myogenin). A definitive diagnosis of pleomorphic malignant peripheral nerve sheath tumor can be difficult unless the pleomorphic sarcoma clearly arises from a benign nerve sheath tumor or arises from a peripheral nerve in a patient with type 1 neurofibromatosis (Figure 5). The only criterion for recognizing extraskeletal osteosarcoma is the production of osteoid or bone by cytologically malignant cells (Figure 6).

As mentioned, several studies have suggested that pleomorphic sarcomas with myogenic differentiated are clinically more aggressive than those without myogenic differentiation. ${ }^{9-11}$ Fletcher et al reviewed 100 cases diagnosed as 'MFH' and concluded (based upon morphology and immunohistochemistry) that 70 of these tumors were non-myogenic pleomorphic sarcomas, whereas 30 showed evidence of myogenic

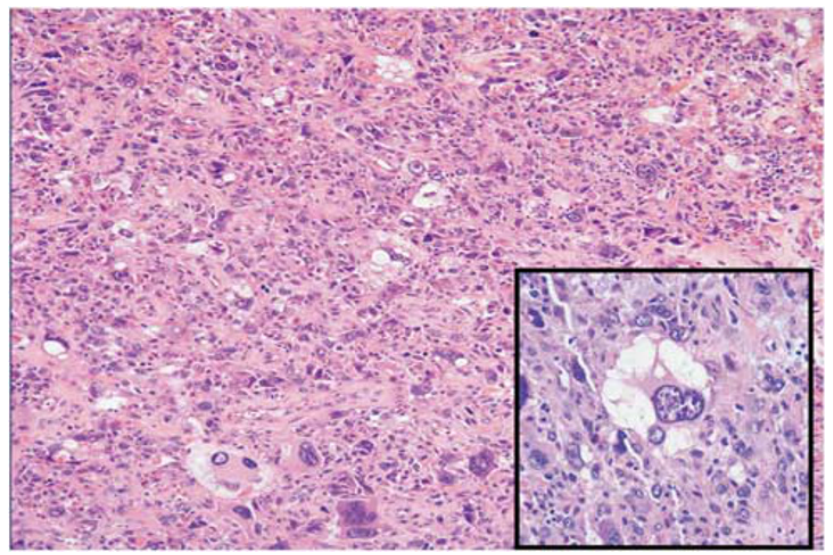

Figure 2 Pleomorphic liposarcoma characterized by the presence of pleomorphic lipoblasts.

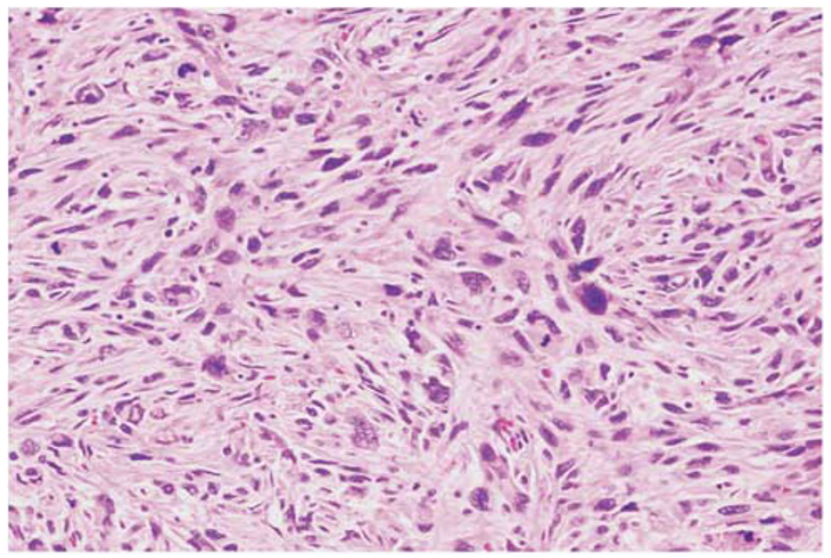

Figure 3 Pleomorphic leiomyosarcoma characterized by pleomorphic spindled cells with eosinophilic cytoplasm. This tumor stains strongly for smooth muscle actin. 
differentiation. ${ }^{10}$ Of these 30 cases, 20 were classified as leiomyosarcoma, 9 as myogenic sarcoma, not otherwise specified, and 1 as rhabdomyosarcoma. The pleomorphic sarcomas with myogenic differentiation were significantly more likely to be deep-seated when compared with those without myogenic differentiation $(83 \%$ versus $63 \%$; $P=0.04$ ), and they were also more likely to be grade 4 tumors (97 versus $67 \% ; P=0.02$ ). Similarly, in 2003, Deyrup et al compared 42 cases of pleomorphic sarcoma with myogenic differentiation to 50 pleomorphic sarcomas without myogenic differentiation. ${ }^{9}$ The only significant difference between these two groups was that the tumors with myogenic differentiation were significantly more likely to be grade 3 sarcomas $(88 \%$ versus $74 \% ; P=0.038$ ). There was a significant difference in overall survival, as those sarcomas with myogenic differentiation had shorter overall survival. Overall survival was also directly correlated with the number of myogenic markers found to be positive, as those with three markers found in a given tumor

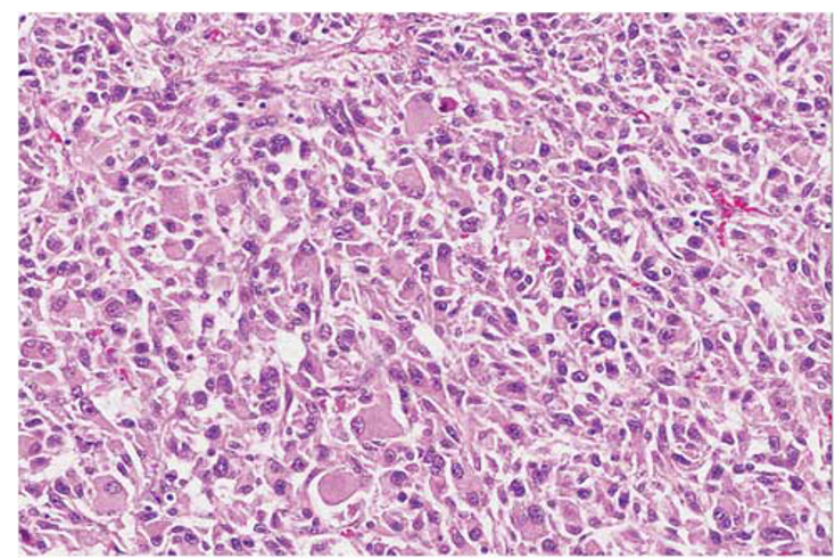

Figure 4 Pleomorphic rhabdomyosarcoma characterized by large pleomorphic cells with cytoplasmic eosinophilia, which stained for myogenin.

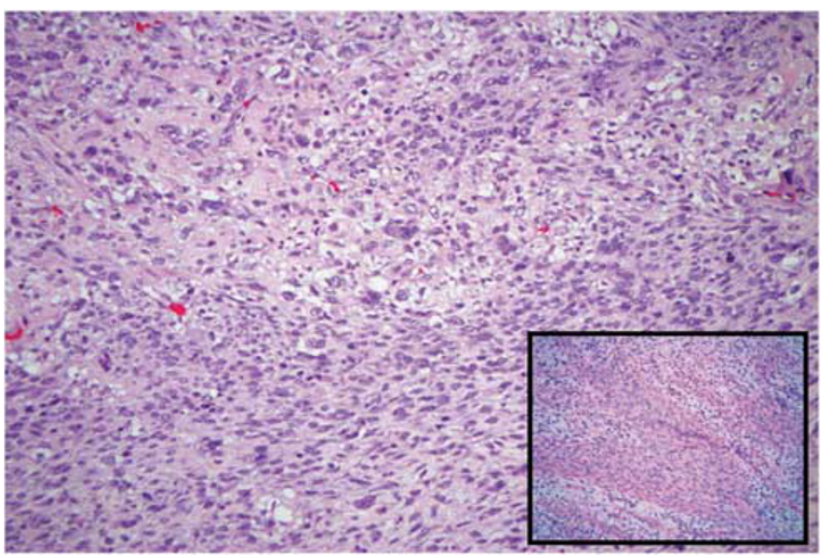

Figure 5 Pleomorphic malignant peripheral nerve sheath tumor that arose in a patient with type 1 neurofibromatosis. The box shows a lower-grade area recognizable as a malignant peripheral nerve sheath tumor. were more aggressive than those with fewer markers. Massi et al evaluated 65 pleomorphic sarcomas of the extremities and compared 31 sarcomas with myogenic differentiation (22 leiomyosarcomas, 4 rhabdomyosarcomas, and 5 myofibrosarcomas) with 34 non-myogenic pleomorphic sarcomas. ${ }^{11}$ The tumors were classified into diagnostic categories based upon morphology, immunohistochemistry, and, in some cases, ultrastructural analysis. Tumor site and myogenic differentiation were both found to be independent predictors of disease relapse for localized tumors by multivariate analysis, but only myogenic differentiation was found to be an independent predictor of overall survival by multivariate analysis.

In summary, there are at least three studies that strongly suggest the adverse prognostic effect of myogenic differentiation in a pleomorphic sarcoma. However, there are a number of practical issues that make the assessment of myogenic differentiation in a pleomorphic sarcoma rather difficult. First, these are relatively uncommon tumors, and there are inconsistencies with regard to diagnostic criteria. There is a relatively small number of cases subjected to multivariate analysis in order to control for tumor site, tumor size, and tumor depth. Exactly how many myogenic markers should be positive to designate a tumor as having myogenic differentiation is unclear, and the extent of staining needed to designate a myogenic marker as positive is similarly unclear.

\section{Pleomorphic sarcoma as a result of dedifferentiation}

The process of tumor progression or dedifferentiation involves the transformation of a low-grade sarcoma to a higher-grade sarcoma, which usually (but not always) resembles a UPS (Figure 7). The most common scenario is the progression of a low-grade well-differentiated liposarcoma to a



Figure 6 Extraskeletal osteosarcoma that arose in the deep soft tissues of the extremity of an older patient. The box in the lower right-hand corner shows malignant cells producing osteoid. 

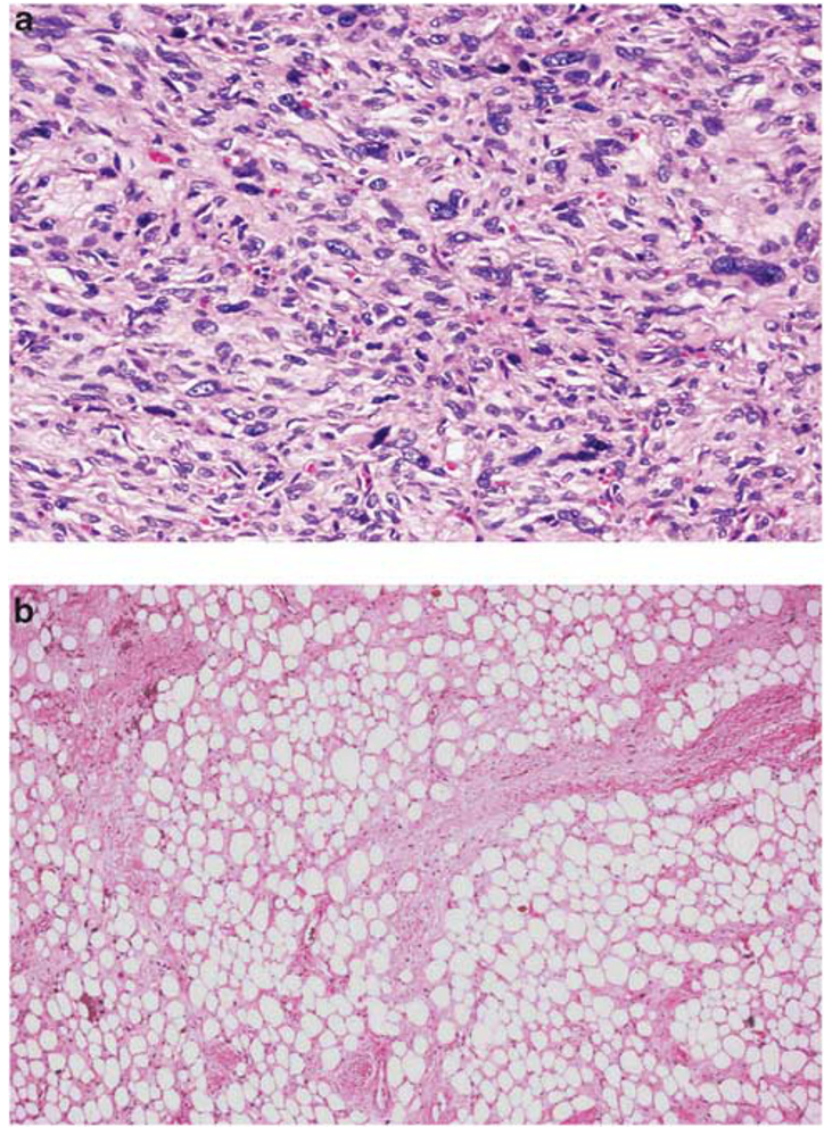

Figure 7 Dedifferentiated liposarcoma. (a) This retroperitoneal mass was comprised predominantly of a high-grade pleomorphic sarcoma without a specific line of differentiation. (b) A minority of the tumor had the histologic appearance of an atypical lipomatous tumor/well-differentiated liposarcoma.

pleomorphic sarcoma (dedifferentiated liposarcoma). Other low-grade neoplasms can also dedifferentiate, including chondrosarcomas, chordomas, and parosteal osteosarcomas. Certainly, in a limited biopsy specimen it can be impossible to prove that a pleomorphic sarcoma is part of a dedifferentiated sarcoma if the low-grade component is not represented. However, in a retroperitoneal sarcoma where dedifferentiated liposarcoma is always a strong consideration, it can often be suggested that the high-grade sarcoma could be part of a dedifferentiated liposarcoma.

There is some evidence to suggest that dedifferentiated sarcomas (dedifferentiated liposarcoma in particular) are more indolent tumors than de novo pleomorphic sarcomas arising in the same location. For example, in the study of 32 cases of dedifferentiated liposarcoma by McCormick et al, 19 of 32 dedifferentiated liposarcomas (59\%) arose in the retroperitoneum or paratesticular region, most of which arose de novo (94\%). ${ }^{12}$ These patients were followed for a mean of 5.6 years, and metastasis was detected in only 4 of 27 cases with follow-up (15\%), a rate much lower than would be expected for de novo pleomorphic sarcomas in that location. Similarly,
Henricks et al studies 155 cases of dedifferentiated liposarcoma, most of which arose in the retroperitoneum, spermatic cord, or scrotum $(77 \%)$ and most of which showed de novo dedifferentiation $(86 \%))^{13}$ Similar to the results reported by McCormick et al, these authors found a metastatic rate of only $18 \%$ for dedifferentiated liposarcomas of the retroperitoneum and an overall metastatic rate of $17 \%$ for dedifferentiated liposarcomas at all sites. Thus, it does appear that dedifferentiated liposarcoma has a lower metastatic rate than other pleomorphic sarcomas arising at the same site. If this is true, then it is logical to presume that it is of clinical importance to distinguish a dedifferentiated liposarcoma from a de novo pleomorphic sarcoma of some other type.

The utility of MDM2 and CDK4 analysis in distinguishing dedifferentiated liposarcoma from other types of pleomorphic sarcoma is controversial, but the preponderance of evidence suggests that this analysis is useful. Inasmuch as both MDM2 and CDK4 are amplified and often immuno-positive in welldifferentiated liposarcomas, it is reasonable to presume that these markers would be similarly expressed in dedifferentiated liposarcomas as the latter are derived from the former. In fact, there are a number of studies that confirm the frequent amplification and immunoexpression of these markers in dedifferentiated liposarcoma. For example, Binh et al found amplification of MDM2 and/or CDK4 in 53 of 55 $(96 \%)$ dedifferentiated liposarcomas, using either RTPCR or a-CGH. ${ }^{14}$ Using these same techniques, however, some of the simulators of dedifferentiated liposarcoma were also found to have amplification of these genes, including myxofibrosarcoma (8/13 cases; $62 \%)$, leiomyosarcoma (5/13 cases; $16 \%)$, MPNST (2/6 cases; 33\%), and 'MFH' (3/39 cases; 8\%). By immunohistochemistry, almost all cases of dedifferentiated liposarcoma expressed MDM2 (52 cases), CDK4 (51 cases), or both markers (49 cases). However, some of the simulators of dedifferentiated liposarcoma also showed immunoexpression of these antigens. For example, 8 of 13 cases of myxofibrosarcoma stained for MDM2, 3 stained for CDK4, and 2 stained for both markers. Thus, although assessment of gene amplification and immunoexpression in dedifferentiated liposarcoma is highly sensitive, it lacks complete specificity. I polled many of my soft tissue pathology colleagues in the United States and in other countries, and it is clear there is a lack of uniformity in the approach to utilizing these techniques in the distinction of dedifferentiated liposarcoma from other types of pleomorphic sarcoma.

\section{Pleomorphic non-mesenchymal neoplasms resembling a pleomorphic sarcoma}

It can be exceedingly difficult to distinguish a UPS from a sarcomatoid carcinoma. A reasonable 
approach would be to assume a pleomorphic malignant neoplasm arising in the skin, mucosal surface, or parenchymal organ is a sarcomatoid carcinoma, until proven otherwise (Figure 8). A battery of epithelial markers including broad-spectrum, low- and high-molecular-weight cytokeratins is required, but equivocal results are not uncommon for several reasons. First, not all sarcomatoid carcinomas show the immunohistochemical expression of epithelial markers. Second, virtually any type of sarcoma, including UPS, can on occasion express cytokeratins. Strong and diffuse cytokeratin expression, especially with multiple antibodies, strongly supports a diagnosis of sarcomatoid carcinoma, as does the recognition of an intraepithelial/ intramucosal dysplastic component. In the end, some cases are not resolvable and can only be diagnosed as a pleomorphic malignant neoplasm, sarcoma versus carcinoma. p63 can also have a role in this immunohistochemical work-up. Although p63 is frequently expressed in a variety of sarcomatoid carcinomas, it is actually very rarely expressed in soft tissue tumors. In the study by Jo and Fletcher in 2011, p63 was expressed in only $9 \%$ of 650 soft
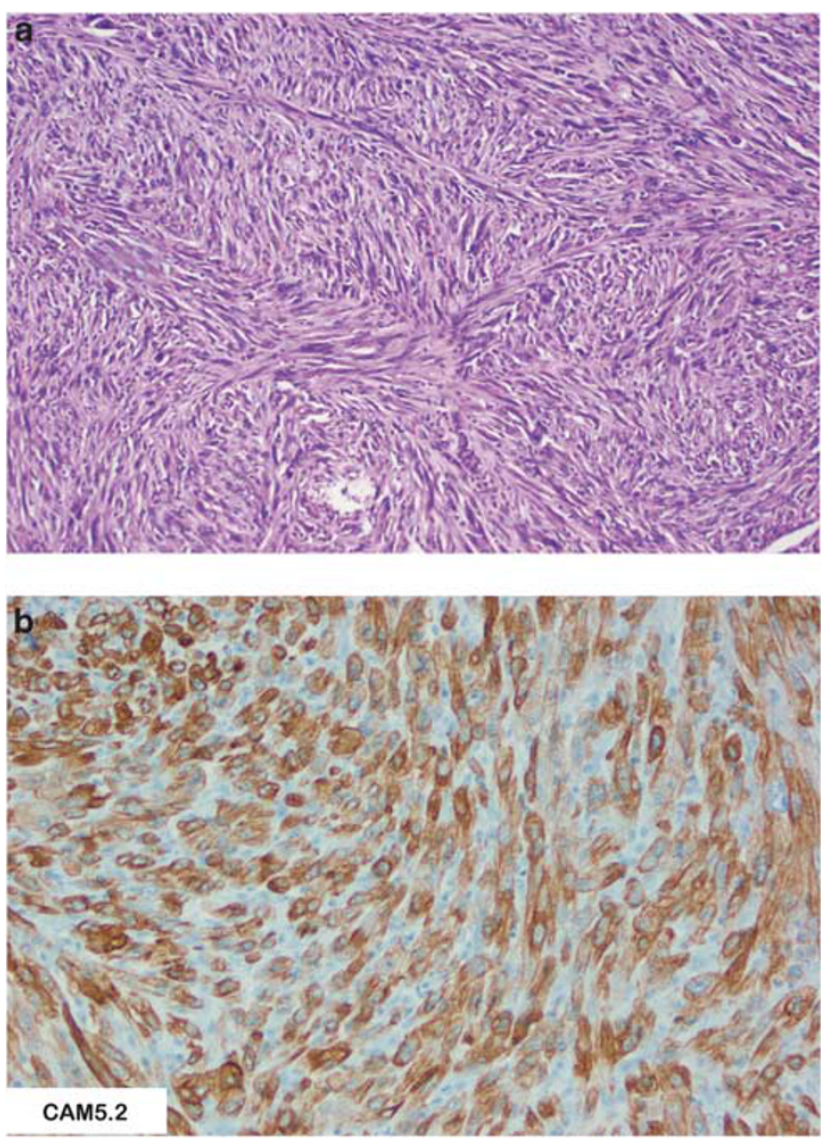

Figure 8 Sarcomatoid carcinoma of the pancreas. (a) This pancreatic mass was composed of a highly cellular proliferation of malignant spindled cells arranged into short intersecting fascicles. (b) Virtually all of the neoplastic cells stain strongly for CAM 5.2, consistent with a sarcomatoid carcinoma. tissue tumors tested. ${ }^{15}$ For example, p63 was found in only 1 of 20 cases of AFX, 0 of 20 cases of dedifferentiated liposarcoma, and 0 of 20 cases of leiomyosarcoma. It should also be kept in mind that sarcomatoid mesothelioma, melanoma, and anaplastic lymphoma can on occasion mimic UPS; a panel of markers including CAM5.2, S-100 protein, melanocytic markers such as HMB45 and Melan A, CD30, and ALK1 can help resolve these issues. Following the exclusion of the aforementioned scenarios, one is left with UPS as a diagnosis of exclusion, the details of which are described below.

\section{UPS: a diagnosis of exclusion}

Depending on definitional criteria, UPS still accounts for a significant proportion of sarcomas occurring in late adult life. It manifests a broad range of histologic appearances, although the most common form consists of a mixture of storiform and pleomorphic areas.

UPS is characteristically a tumor of late adult life, with most cases occurring in persons between the ages of 50 and 70 years. ${ }^{16}$ Tumors in children are exceedingly rare, and this diagnosis should always be made with caution in patients under 20 years of age. Approximately two-thirds occur in men, and whites are affected more often than blacks or Asians. The tumor occurs most frequently in the lower extremity, especially the thigh, followed by the upper extremity, usually as a painless, slowly enlarging mass. As mentioned previously, careful sampling and microscopic observation are necessary in retroperitoneal lesions to exclude dedifferentiated liposarcoma, a lesion which in our experience is far more common than de novo UPS in this location.

Microscopically, the classic form of UPS has a highly variable morphologic pattern and shows frequent transitions from storiform to pleomorphic areas, although the emphasis in most tumors is on haphazardly arranged pleomorphic zones. Storiform areas consist of plump spindle cells arranged in short fascicles in a cartwheel, or storiform, pattern around slit-like vessels. Although such tumors resemble dermatofibrosarcoma protuberans, they differ by a less distinctive storiform pattern and by the presence of occasional plump histiocytic cells, numerous typical and atypical mitotic figures, and secondary elements including xanthoma cells and chronic inflammatory cells. Although this pattern is easily recognized, it is seldom seen throughout the entire tumor. Instead, most tumors have a combination of storiform and pleomorphic areas, with a preponderance on the latter. Least often, tumors have a fascicular growth pattern and resemble fibrosarcomas, except for scattered giant cells, and it may be an arbitrary distinction in some cases as to whether a given tumor should be designated as a UPS or fibrosarcoma. In contrast to the storiform 
areas, pleomorphic areas contain plumper fibroblastic cells and more rounded histiocyte-like cells arranged haphazardly with no particular orientation to vessels. Pleomorphism and mitotic activity are usually more prominent.

The stroma and secondary elements vary considerably in the storiform and pleomorphic areas. Usually, the stroma consists of delicate collagen fibrils encircling individual cells but occasionally collagen deposition is extensive and widely separates cells. Rarely, the stroma contains metaplastic osteoid or chondroid material. If, however, bone or cartilage is extensive and/or appears immature, the tumor should be classified as an osteo- or chondrosarcoma. The vasculature, although elaborate, is seldom appreciated unless it becomes dilated and resembles that of a hemangiopericytoma.

Some examples of this tumor have numerous giant cells, a lesion formerly referred to as the giant cell type of MFH or malignant giant cell tumor of soft parts. ${ }^{17,18}$ These tumors tend to be distinctly multinodular and composed of a mixture of spindled, rounded, and osteoclast-type giant cells. Dense fibrous bands containing vessels often encircle the nodules, which frequently show secondary hemorrhage and necrosis. The cells display pleomorphism and prominent mitotic activity, and may contain ingested material such as lipid or hemosiderin. The nuclei of the osteoclast-type giant cells tend also to be of high nuclear grade. Focal osteoid or mature bone is present in up to $50 \%$ of these cases and is usually located at the periphery of the tumor nodules.

\section{Immunohistochemical findings}

The role of immunohistochemistry in the diagnosis of UPS has traditionally been an ancillary one, primarily serving as a means to exclude other pleomorphic tumors. Thus, the diagnosis continues to presuppose thorough sampling and evaluation of hematoxylin-eosin-stained sections. Despite the limited diagnostic applications of immunohistochemistry aside from excluding other lesions, there is ample evidence that these tumors do not display features of monocytes or macrophages but, rather, fibroblasts/myofibroblasts. ${ }^{8,19,20}$ Many of these tumors show focal immunoreactivity for smooth muscle actin, but stains for desmin and h-caldesmon are typically negative. ${ }^{21}$ Some examples also show rare cytokeratin-positive cells, which can cause confusion with those tumors in which a sarcomatoid carcinoma is a real consideration. ${ }^{22-24}$ Thus, focal immunoreactivity for any number of intermediate filaments is, in my opinion, insufficient evidence of a specific line of differentiation and should not dissuade one from rendering a diagnosis of UPS. On the other hand, diffuse immunoreactivity is far more likely to reflect a specific line of differentiation.

\section{Cytogenetic and molecular genetic findings}

Over the past several decades, studies have reported a variety of cytogenetic abnormalities in ' $\mathrm{MFH}$ ' but of course the utility of this information is limited by the varying criteria for making this diagnosis. ${ }^{25,26} \mathrm{In}$ general, pleomorphic sarcomas of all types are characterized by complex but nonspecific cytogenetic aberrations and, as such, this technique is not useful in distinguishing among these pleomorphic sarcomas. ${ }^{27}$

More recently, a number of studies using comparative genomic hybridization have evaluated UPS and compared the findings to those of other pleomorphic sarcomas. ${ }^{28-31}$ Interestingly, several studies have found striking similarities between UPS and pleomorphic leiomyosarcoma suggesting a shared lineage. ${ }^{29,31}$

\section{Clinical behavior}

The vast majority of UPS are high-grade lesions having a local recurrence rate ranging from 19-31\%, a metastatic rate of $31-35 \%$, and a 5-year survival of $65-70 \% .^{32-37}$ Both local recurrence and distant metastases often develop within 12-24 months of diagnosis. Only a minority of patients develop metastases after 5 years, with the common metastatic sites being lung $(90 \%)$, bone $(8 \%)$, and liver (1\%). Regional lymph node metastases are decidedly uncommon.

The factors that correlate consistently with metastasis, survival, or both are depth, tumor size, grade, necrosis, and local recurrence, although they are not necessarily independent variables. For example, size and depth appear to co-vary because large tumors tend to be deep tumors. In the study by Engellau et al, necrosis and local recurrence were significant predictors of metastasis within the first 2 years of diagnosis and throughout a longitudinal follow-up period, whereas only tumor depth and local recurrence were significant predictors beyond 2 years. ${ }^{32}$

\section{Summary}

In summary, pleomorphic malignant neoplasms are commonly encountered by surgical pathologists. Although many of these will ultimately be diagnosed as a pleomorphic sarcoma, close morphologic observation and judicious use of ancillary techniques may help precisely classify the tumor as some type of pleomorphic sarcoma with a specific line of differentiation. Still others, particularly those located in the retroperitoneum, represent dedifferentiated sarcomas (mostly commonly dedifferentiated liposarcoma). Most important, one must always be cognizant of the possibility that one is 
dealing with a pseudosarcoma, most commonly a sarcomatoid carcinoma, particularly when encountering a pleomorphic malignant neoplasm of the skin, mucosal surface, or parenchymal organ.

\section{Disclosure/conflict of interest}

The author declares no conflict of interest.

\section{References}

1 Ozzello L, Stout AP, Murray MR. Cultural characteristics of malignant histiocytomas and fibrous xanthomas. Cancer 1963;16:331-344.

2 O'Brien JE, Stout AP. Malignant fibrous xanthomas. Cancer 1964;17:1445-1455.

3 Iwasaki $\mathrm{H}$, Isayama $\mathrm{T}$, Johzaki $\mathrm{H}$, et al. Malignant fibrous histiocytoma. Evidence of perivascular mesenchymal cell origin immunocytochemical studies with monoclonal anti-MFH antibodies. Am. J. Pathol 1987;128:528-537.

4 Iwasaki $\mathrm{H}$, Isayama $\mathrm{T}$, Ohjimi $\mathrm{Y}$, et al. Malignant fibrous histiocytoma. A tumor of facultative histiocytes showing mesenchymal differentiation in cultured cell lines. Cancer 1992;69:437-447.

5 Roholl PJ, Kleyne J, Elbers H, et al. Characterization of tumour cells in malignant fibrous histiocytomas and other soft tissue tumours in comparison with malignant histiocytes. I. Immunohistochemical study on paraffin sections. J Pathol 1985;147:87-95.

6 Roholl PJ, Kleyne J, Van Unnik JA. Characterization of tumor cells in malignant fibrous histiocytomas and other soft-tissue tumors, in comparison with malignant histiocytes. II. Immunoperoxidase study on cryostat sections. Am J Pathol 1985;121:269-274.

7 Fletcher CD. Pleomorphic malignant fibrous histiocytoma: fact or fiction? A critical reappraisal based on 159 tumors diagnosed as pleomorphic sarcoma. Am J Surg Pathol 1992;16:213-228.

8 Montgomery E, Fisher C. Myofibroblastic differentiation in malignant fibrous histiocytoma (pleomorphic myofibrosarcoma): a clinicopathological study. Histopathology 2001;38:499-509.

9 Deyrup AT, Haydon RC, Huo D, et al. Myoid differentiation and prognosis in adult pleomorphic sarcomas of the extremity: an analysis of 92 cases. Cancer 2003;98:805-813.

10 Fletcher CD, Gustafson P, Rydholm A, et al. Clinicopathologic re-evaluation of 100 malignant fibrous histiocytomas: prognostic relevance of subclassification. J Clin Oncol 2001;19:3045-3050.

11 Massi D, Beltrami G, Capanna R, et al. Histopathological re-classification of extremity pleomorphic soft tissue sarcoma has clinical relevance. Eur J Surg Oncol 2004;30:1131-1136.

12 McCormick D, Mentzel T, Beham A, et al. Dedifferentiated liposarcoma. Clinicopathologic analysis of 32 cases suggesting a better prognostic subgroup among pleomorphic sarcomas. Am J Surg Pathol 1994;18: 1213-1223.

13 Henricks WH, Chu YC, Goldblum JR, et al. Dedifferentiated liposarcoma: a clinicopathological analysis of 155 cases with a proposal for an expanded definition of dedifferentiation. Am J Surg Pathol 1997;21: 271-281.

14 Binh MBN, Sastre-Garau X, Guillou L, et al. MDM2 and CDK4 immunostainings are useful adjuncts in diagnosing well-differentiated and dedifferentiated liposarcoma subtypes: a comparative analysis of 559 soft tissue neoplasms with genetic data. Am J Surg Pathol 2005;29:1340-1347.

15 Jo VY. Fletcher CDM. p63 immunohistochemical staining is limited in soft tissue tumors. Am J Clin Pathol 2011;136:762-766.

16 Weiss SW, Enzinger FM. Malignant fibrous histiocytoma: an analysis of 200 cases. Cancer 1978;41: 2250-2266.

17 Guccion JG, Enzinger FM. Malignant giant cell tumor of soft parts. An analysis of 32 cases. Cancer 1972;29:1518-1529.

18 van Haelst UJ, de Haas van Dorsser AH. Giant cell tumor of soft parts. An ultrastructural study. Virchows Arch A Pathol Anat Histol 1976;371:199-217.

19 Brecher ME, Franklin WA. Absence of mononuclear phagocyte antigens in malignant fibrous histiocytoma. Am J Clin Pathol 1986;86:344-348.

20 Wood GS, Beckstead JH, Turner RR, et al. Malignant fibrous histiocytoma tumor cells resemble fibroblasts. Am J Surg Pathol 1986;10:323-335.

21 Agaimy A, Gaumann A, Schroeder J, et al. Primary and metastatic high-grade pleomorphic sarcoma/malignant fibrous histiocytoma of the gastrointestinal tract: an approach to the differential diagnosis in a series of five cases with emphasis on myofibroblastic differentiation. Virchows Arch 2007;451:949-957.

22 Lawson CW, Fisher C, Gatter KC. An immunohistochemical study of differentiation in malignant fibrous histiocytoma. Histopathology 1987;11:375-383.

23 Litzky LA, Brooks JJ. Cytokeratin immunoreactivity in malignant fibrous histiocytoma and spindle cell tumors: comparison between frozen and paraffinembedded tissues. Mod Pathol 1992;5:30-34.

24 Weiss SW, Bratthauer GL, Morris PA. Postirradiation malignant fibrous histiocytoma expressing cytokeratin. Implications for the immunodiagnosis of sarcomas. Am J Surg Pathol 1988;12:554-558.

25 Gazziola C, Cordani N, Wasserman B, et al. Malignant fibrous histiocytoma: a proposed cellular origin and identification of its characterizing gene transcripts. Int J Oncol 2003;23:343-351.

26 Al-Agha OM, Igbokwe AA. Malignant fibrous histiocytoma: between the past and the present. Arch Pathol Lab Med 2008;132:1030-1035.

27 Mertens F, Fletcher CD, Dal Cin P, et al. Cytogenetic analysis of 46 pleomorphic soft tissue sarcomas and correlation with morphologic and clinical features: a report of the CHAMP Study Group. Chromosomes and MorPhology. Genes Chromosomes Cancer 1998;22: 16-25.

28 Kageyama K, Moriyama T, Hizuka N, et al. Hypoglycemia associated with big insulin-like growth factor II produced during development of malignant fibrous histiocytoma. Endocr J 2003;50:753-758.

29 Larramendy ML, Gentile M, Soloneski S, et al. Does comparative genomic hybridization reveal distinct differences in DNA copy number sequence patterns between leiomyosarcoma and malignant fibrous histiocytoma? Cancer Genet Cytogenet 2008;187:1-11.

30 Nishio J, Iwasaki H, Nabeshima K, et al. Establishment of a new human pleomorphic malignant fibrous 
histiocytoma cell line, FU-MFH-2: molecular cytogenetic characterization by multicolor fluorescence in situ hybridization and comparative genomic hybridization. J Exp Clin Cancer Res 2010;29:153.

31 Carneiro A, Francis P, Bendahl P-O, et al. Indistinguishable genomic profiles and shared prognostic markers in undifferentiated pleomorphic sarcoma and leiomyosarcoma: different sides of a single coin? Lab Invest 2009;89:668-675.

32 Engellau J, Anderson H, Rydholm A, et al. Time dependence of prognostic factors for patients with soft tissue sarcoma: a Scandinavian Sarcoma Group Study of 338 malignant fibrous histiocytomas. Cancer 2004;100:2233-2239.

33 Le Doussal V, Coindre JM, Leroux A, et al. Prognostic factors for patients with localized primary malignant fibrous histiocytoma: a multicenter study of 216 patients with multivariate analysis. Cancer 1996;77: 1823-1830.

34 Salo JC, Lewis JJ, Woodruff JM, et al. Malignant fibrous histiocytoma of the extremity. Cancer 1999;85: 1765-1772.

35 Gibbs JF, Huang PP, Lee RJ, et al. Malignant fibrous histiocytoma: an institutional review. Cancer Invest 2001;19:23-27.

36 Zagars GK, Mullen JR, Pollack A. Malignant fibrous histiocytoma: outcome and prognostic factors following conservation surgery and radiotherapy. Int J Radiat Oncol Biol Phys 1996;34:983-994.

37 Belal A, Kandil A, Allam A, et al. Malignant fibrous histiocytoma: a retrospective study of 109 cases. Am J Clin Oncol 2002;25:16-22. 